Check for updates

Cite this: RSC Adv., 2018, 8, 31372

\title{
Polypeptide - decorated nanoliposomes as novel delivery systems for lutein
}

\author{
Yan Jiao, (D) ab Dajing Li, ${ }^{* a}$ Chunquan Liu, ${ }^{\text {a }}$ Ying Chang, ${ }^{\text {b Jiangfeng Song }}{ }^{\text {a }}$ \\ and Yadong Xiao ${ }^{a}$
}

Lutein (LUT) is a bioactive food compound found in various vegetables and plays a critical role in the promotion of health and well-being. However, lutein is an unstable molecule which has a very low bioavailability caused by its poor solubility in aqueous media, and is poorly absorbed when administered orally. To enhance the stability, release and bioactivity of lutein, poly-L-lysine (PLL) decorated nanoliposomes (PLL-LUT-NLP) were developed as novel delivery systems for lutein. The mean particle size of PLL-LUT-NLP was found to be in the range 264-367 nm with a low polydispersity index (PDI < 0.4). The zeta potential changed from $-38.6 \mathrm{mV}$ in undecorated nanoliposomes to $-27.9 \mathrm{mV}$ in PLLdecorated nanoliposomes. Furthermore, the lutein entrapment efficiency (EE\%) of PLL-LUT-NLP was found to be highest in nanoliposomes decorated with $0.06 \%(\mathrm{w} / \mathrm{v})$ PLL. PLL could protect lutein in nanoliposomes from degradation and promote the lutein release from the nanoliposomes in gastrointestinal fluid conditions. Additionally, the PLL-decorated nanoliposomes maintained the antioxidant activity of the lutein, and the antiproliferative activity was more significant than that of undecorated nanoliposomes in inhibiting the proliferation of human tumor cells. These results suggest that PLL-decorated nanoliposomes have potential to be used for efficient delivery of lutein and further improve its bioavailability.

Received 9th July 2018

Accepted 27th August 2018

DOI: $10.1039 / \mathrm{c} 8 \mathrm{ra05838e}$

rsc.li/rsc-advances particularly lutein and lycopene in small doses may potentially prevent colon carcinogenesis. ${ }^{9}$

Despite its numerous biological benefits, lutein is an unstable molecule of conjugated carbon-carbon double-bonds with a low absorption rate after administration and poor bioavailability due to its insolubility in the aqueous media. To enhance solubility, stability and bioavailability of lutein, extensive strategies have been developed for its encapsulation by suitable carriers, including nanoparticles, nanoencapsulation and nanoliposome. ${ }^{10,11}$ For example, lutein entrapped in chitosan/PLGA nanoparticles displayed a higher bioavailability than unmodified lutein in mice. ${ }^{12}$ The solubility of lutein could be improved by chitosan (CS)/poly-glutamic acid (PGA) nanoencapsulation. ${ }^{13}$

Nanoliposomes, spherical bilayer vesicles from dispersion of polar lipids in aqueous solvents, have been widely studied for many advantages. The technology of nanoliposomes can offer a novel approach for enhancing bioavailability of fat-soluble and unstable nutrients. ${ }^{14}$ They enhance bioactive agent performance by improving the solubility and bioavailability, in vitro and in vivo stability, as well as preventing the unwanted interactions with other molecules. ${ }^{15}$ In order to improve absorption and bioavailability of lutein, nanoliposomes are also one of the most extensively investigated delivery systems for encapsulation for protecting and controlling release of lutein. ${ }^{16,17}$ anstitute of Farm Products Processing, Jiangsu Academy of Agricultural Sciences, Nanjing 210014, China. E-mail: jiaoyan_3000@126.com; lidajing@163.com

${ }^{b}$ College of Food and Biological Engineering, Qiqihar University, Qiqihar 161006, China 
However, the practical application of nanoliposomes is limited by their low biological activity, due to the oxidative decomposition during long-term storage, digestion under low $\mathrm{pH}$ and enzymatic condition, and the weak release and absorption in the intestinal condition. ${ }^{18}$ Surface-decorated liposomes using functional cationic polymers to improve the stability and absorption of loaded active ingredients have been developed. ${ }^{19,20}$ Poly-L-lysine (PLL), a cationic polypeptide, has been shown to enhance the cellular uptake and bioactivity of drugs by increasing the permeability of various compounds as a modifier for active ingredients and drug nano-carriers. ${ }^{21}$

Microencapsules, based on decoration of poly-L-lysine (PLL) on the nanoparticles and liposomal surface, are an effective way for reducing the damage of liposomal membrane and the leakage of encapsulated compounds to improve encapsulation efficiency and bioactivity. ${ }^{22,23}$ Importantly, liposome surface modification with PLL significantly increased delivery of the marker coumarin-6, which had high biocompatibility and low toxicity in cells of the retina segment. ${ }^{24}$ PLL decoration could effectively penetrate and overcome biological barriers to cellular and tissue uptake and enhance the absorption effects in intestine. ${ }^{25,26}$

Hence, the present study focused primarily on the preparation of nanoliposomes and decoration with PLL for encapsulation of lutein. The physicochemical characteristics of the samples and their structural property were determined using dynamic light scattering (DLS), transmission electron microscopy (TEM) and fourier transform infrared (FTIR) spectroscopy. In addition, the encapsulation efficiency (EE), in vitro stability and release rate were studied. The antioxidant (DPPH, ABTS assay) and anticancer activity of free lutein, lutein nanoliposomes and PLL-decorated nanoliposomes were also investigated.

\section{Materials and methods}

\subsection{Materials}

Lutein (98\%) and lecithin were purchased from Shanghai Yuanye Biotechnology Co., Ltd. (Shanghai, China) and Beijing Solarbio Science \& Technology Co., Ltd. (Beijing, China), respectively. PolyL-lysine, cholesterol (MKBF1936 V), 1,1-diphenyl-2-picrylhydrazyl radical 2,2-diphenyl-1-(2,4,6-trinitrophenyl)hydrazyl (DPPH) and 2,2'-azino-bis(3-ethylbenzothiazoline-6-sulfonic acid) (ABTS) were from Sigma-Aldrich Chemical Co. (St. Louis, MO, USA). Ethanol, Tween 80, methanol, and other reagent chemicals were all of analytical grade.

\subsection{Preparation of PLL decorated nanoliposomes}

Lutein nanoliposomes (LUT-NLP) were produced by an ethanol injection method, as described in a previous study. ${ }^{27}$ Lutein, cholesterol, Tween 80 , and lecithin were mixed at a mass ratio of $1: 10: 40: 10$. The mixture $\left(1.0 \mathrm{mg} \mathrm{mL}^{-1}\right.$ lutein, the lecithin concentration was kept at $10 \mathrm{mg} \mathrm{mL}^{-1}$ ) was well dissolved in absolute ethanol and quickly injected into the same volume of phosphate-buffered saline (PBS, pH 6.0, $0.05 \mathrm{M}$ ). It was stirred vigorously and kept for half an hour at $50{ }^{\circ} \mathrm{C}$. The liposomal system was transferred to a rotary evaporator and incubated in a $50{ }^{\circ} \mathrm{C}$ water bath. The LUT-NLP (Fig. 1A) were obtained when ethanol was removed under a reduced pressure during the hydration process.

Surface decoration of prepared LUT-NLP was accomplished by mixing LUT-NLP suspension with an equal volume of the PLL (Fig. 1B) solution. An equal volume of PLL dispersion in PBS was added into the LUT-NLP suspension, the final concentration of PLL was adjusted from 0.04 to $0.08 \mathrm{mg} \mathrm{mL}^{-1}$. Then, the liposomal system was incubated at $50{ }^{\circ} \mathrm{C}$ for $120 \mathrm{~min}$ with subsequent cooling at room temperature to produce PLL-LUT-NLP (Fig. 1C). The final samples were filled into vials and stored at the same conditions as bare liposomes. ${ }^{28}$

\subsection{Encapsulation efficiency of lutein}

The encapsulation efficiency of lutein in the nanoliposomes was calculated by the difference between total amount of lutein used to prepare the loaded systems and the remaining amount of free lutein in aqueous medium. ${ }^{29}$ Free lutein was extracted to determine its amount as follows: $1.0 \mathrm{~mL}$ of nanoliposomes and $3 \mathrm{~mL}$ of petroleum ether were mixed by vortexing vigorously for $5 \mathrm{~min}$ at ambient temperature. The mixed sample was centrifuged at $2000 \mathrm{rpm}$ for $5 \mathrm{~min}$ and the supernatant was collected in a tube. The operation procedure was repeated thrice.

The supernatant was diluted to $10 \mathrm{~mL}$ with petroleum ether. Amount of free lutein was assayed for lutein content by HPLC at
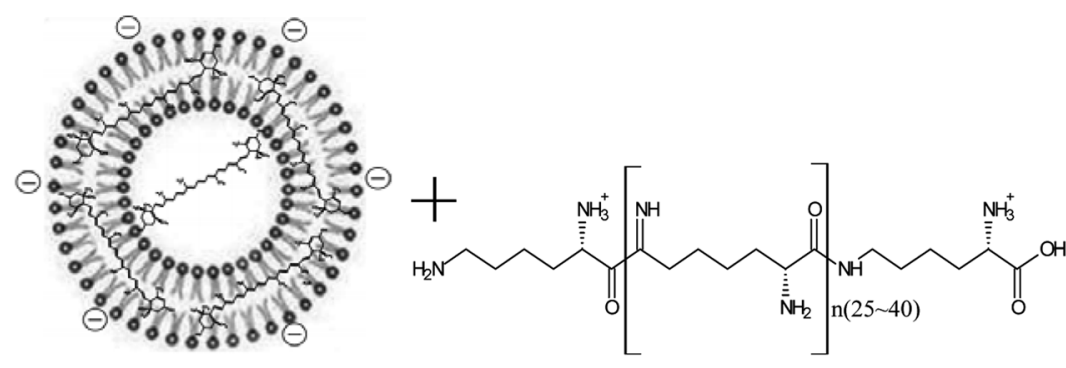

B ( Poly-L-lysine )

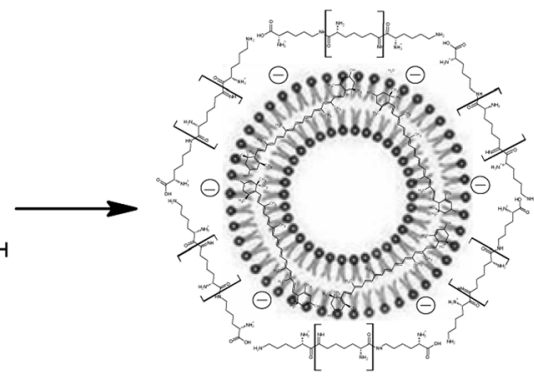

C ( PLL-LUT-NLP)

Fig. 1 Formation mechanism of PLL decorated lutein nanoliposome. 
$445 \mathrm{~nm}$ (Agilent Technologies, Germany; column: $\mathrm{YMC}^{-\mathrm{C}_{30}}$ ) as described in our previous study. ${ }^{30}$ A calibration curve was made with solutions of lutein at concentrations from 5 to $100 \mu \mathrm{g}$ $\mathrm{mL}^{-1}$. Each sample was assayed in triplicate. The entrapment efficiency (EE\%) was calculated by the following formula:

$$
\mathrm{EE}(\%)=\left[1-\left(W_{\text {free }} / W_{\text {total }}\right)\right] \times 100
$$

$W_{\text {free }}$ and $W_{\text {total }}$ were the weight of free lutein and initial weight of lutein added in the preparation, respectively.

\subsection{Particle size and zeta potential analysis}

Liposomal dispersions $(1.0 \mathrm{~mL})$ were suspended in $100 \mathrm{~mL}$ of phosphate buffer and taken into polystyrene cuvettes. Both zaverage diameter $\left(D_{\mathrm{z}}\right)$ and zeta $(\zeta)$ potential were measured using a Nano-ZS90 particle size analyzer (Malvern Instruments Ltd., Malvern, UK). ${ }^{31}$ Each sample was analyzed thrice.

\subsection{Transmission electron microscopy (TEM)}

Morphological observation of nanoliposomes was performed by TEM (H-7000, Hitachi, Japan). TEM was performed to visualize the morphology of LUT-NLP and PLL-LUT-NLP. A small droplet of nanoliposomal suspension was deposited on a copper grid and allowed to air dry for $5 \mathrm{~min}$. The grid was then negatively stained with $2 \%$ phosphotungstic acid for $1 \mathrm{~min}$. Subsequently, the excess liquid was removed with a filter paper. After air drying at room temperature, the sample was observed under the TEM. $^{32}$

\subsection{FT-IR assay}

An appropriate amount of $\mathrm{KBr}$ was dried under an infrared lamp, mixed with the freeze-dried LUT-NLP, PLL and PLL-LUTNLP, and ground into a plate. The plate was scanned by an infrared spectrometer (Spectrum 100, Perkin Elmer, USA) from 4000 to $550 \mathrm{~cm}^{-1}$. The infrared spectra of PLL-LUT-NLP were recorded and analyzed in comparison with LUT-NLP. Infrared spectra of PLL and PLL-LUT-NLP were compared to further verify whether PLL was decorated in LUT-NLP. ${ }^{33}$

\subsection{Stability evaluation of lutein in nanoliposome during in vitro digestion}

In vitro digestion of LUT-NLP and PLL-LUT-NLP was carried out in simulated gastric fluid (SGF) and simulated intestinal fluid (SIF) separately according to the method of Davidov-Pardo et al. with a slight modification. ${ }^{34}$ By taking LUT as a control group, $5 \mathrm{~mL}$ of LUT, LUT-NLP and PLL-LUT-NLP solution was mixed with $25 \mathrm{~mL}$ SGF or SIF. The final concentration of LUT present in the suspension was $200 \mu \mathrm{g} \mathrm{mL}{ }^{-1}$. The suspensions were incubated in a shaking water bath (100 rpm) at $37{ }^{\circ} \mathrm{C}$ and sampled after $0,2,4,6,8,10$ and $12 \mathrm{~h}, 1.0 \mathrm{~mL}$ suspensions were sonicated in the presence of $3 \mathrm{~mL}$ ethanol, the mixed sample was centrifuged at $2000 \mathrm{rpm}$ for $5 \mathrm{~min}$ and the supernatant was collected. The supernatant was subsequently assayed for residual LUT content using HPLC assay as described above.

SGF and SIF were prepared according to Frenzel et al. with a slight modification. ${ }^{35} \mathrm{SGF}$ was produced by mixing $25 \mathrm{~mL}$ of distilled water with a $\mathrm{pH}$ value set to 2.0 with hydrochloric acid and $1.0 \mathrm{~mL}$ of $0.4 \%$ pepsin solution $\left(800-2000 \mathrm{U} \mathrm{mg}^{-1}\right.$ of protein). SIF contained sodium hydroxide $\left(1.81 \mathrm{~g} \mathrm{~L}^{-1}\right)$, potassium dihydrogen phosphate $\left(8.09 \mathrm{~g} \mathrm{~L}^{-1}\right)$, pancreatin $(4.76 \mathrm{~g}$ $\left.\mathrm{L}^{-1}\right)$, and bile salts $\left(5.16 \mathrm{~g} \mathrm{~L}^{-1}\right)$.

Before in vitro digestion of LUT-NLP and PLL-LUT-NLP, the SGF and SIF were incubated at $37^{\circ} \mathrm{C}$ for preheating in the water bath. HPLC assay of LUT before and after incubation in SGF and SIF was determined to further evaluate the detailed protective effect of nanoliposome decorated with PLL on the degradation rate of LUT. The degradation rate was calculated by the following equation.

$$
D(\%)=\left[\left(C_{0}-C_{t}\right) / C_{0}\right] \times 100
$$

where $D$ was degradation rate, and $C_{0}$ and $C_{t}$ were the lutein concentration before in vitro digestion and residual LUT concentration, respectively.

\subsection{In vitro release of lutein in nanoliposome}

In vitro release of lutein from LUT-NLP and PLL-LUT-NLP was carried out in SGF and SIF separately according to the previous study, with a slight modification. ${ }^{36} 10 \mathrm{~mL}$ of each nanoliposomal solution was taken in a dialysis bag with a cut off size of $10 \mathrm{kDa}$. The tube was immersed in $100 \mathrm{~mL}$ SGF and SIF respectively, thermostated at $37.0 \pm 0.5^{\circ} \mathrm{C}$. The fluid outside the dialysis bag was stirred at $120 \mathrm{rpm}$ and sampled after $0,2,4,6$, $8,10,12$ and $20 \mathrm{~h}$, respectively. Samples $(1.0 \mathrm{~mL})$ were taken to determine the lutein content at a certain time by HPLC method mentioned previously. In addition, equal volume of fresh dissolution medium was added into the tube to maintain the constant volume. The mean calculated values were obtained with 3 replicates. The release rate was calculated by the following equation.

$$
Q(\%)=\left(M_{n} / M\right) \times 100
$$

where $Q$ was the lutein release rate, $M_{n}$ and $M$ were the lutein content at a certain time $n$ and the lutein content initially entrapped in the nanoliposomes, respectively.

\subsection{Antioxidant activities}

2.9.1. DPPH radical scavenging activity assay. DPPH radical scavenging activity of the lutein, LUT-NLP and PLL-LUTNLP samples were determined as previously described with a few modifications. ${ }^{37}$ Briefly, $2.0 \mathrm{~mL}$ of the sample was mixed with $2.0 \mathrm{~mL}$ of $0.16 \mathrm{mM}$ DPPH dissolved in ethanol. The mixture was mixed vigorously and then kept for $30 \mathrm{~min}$ in the dark at room temperature. The absorbance of the resulting solution was recorded at $517 \mathrm{~nm}$. A blank was prepared in the same manner except that ethanol was used instead of the sample. The scavenging activity was calculated as:

$$
\begin{gathered}
\text { DPPH scavenging activity }(\%)= \\
{[(\text { blank absorbance }- \text { sample absorbance }) /} \\
\text { blank absorbance }] \times 100
\end{gathered}
$$


2.9.2. ABTS radical scavenging activity. ABTS radical scavenging capacity was evaluated using the modified procedure proposed by Ramezanzade et al. ${ }^{38}$ The stock solutions of ABTS radical consisting of $7.0 \mathrm{mM}$ ABTS in $2.45 \mathrm{mM}$ potassium persulfate were stored in the dark at room temperature for $16 \mathrm{~h}$. An aliquot of stock solution was diluted with distilled water to prepare a working ABTS solution with an absorbance of $0.7 \pm$ 0.02 at $734 \mathrm{~nm}$. An aliquot $(100 \mu \mathrm{L})$ of the sample was mixed with $3.0 \mathrm{~mL}$ of the ABTS reagent and absorbance was measured at $734 \mathrm{~nm}$ after reacting with fresh ABTS solution for $10 \mathrm{~min}$ in the dark. The ABTS scavenging activity of the samples was calculated from eqn (5):

ABTS scavenging activity $(\%)=$

$$
\left[\left(A_{\text {blank }}-A_{\text {sample }}\right) / A_{\text {blank }}\right] \times 100
$$

where, $A_{\text {blank }}$ is the absorbance without sample at $734 \mathrm{~nm}$, and $A_{\text {sample }}$ is the absorbance of the sample solution.

\subsection{Cell culture}

The Caco-2 cells (CBCAS, Shanghai, China) were maintained in Dulbecco's modified Eagle's medium (DMEM) (Hyclone Laboratories, Inc., USA) and supplemented with 10\% fatal bovine serum (FBS) (Gibco BRL Co., Ltd., USA), penicillin (100 kU L ${ }^{-1}$ ), and streptomycin $\left(100 \mathrm{~g} \mathrm{~L}^{-1}\right)$ at $37{ }^{\circ} \mathrm{C}$ in a $5 \% \mathrm{CO}_{2}$ atmosphere in a humidified incubator. After reaching $70-80 \%$ confluence, the cells were sub-cultured and maintained with medium changes every 1-2 days.

\subsection{Cell proliferation}

Cell proliferation was tested by the MTT assay. ${ }^{39}$ The Caco-2 cells were prepared and dispersed in 96-well cell culture plates at a cellular density of $1.0 \times 10^{5}$ cells per well. After incubating with different concentrations of lutein, LUT-NLP and PLL-LUT-NLP for $24 \mathrm{~h}, 0.020 \mathrm{~mL}$ of MTT solution $(2 \mathrm{mg}$ $\mathrm{mL}^{-1}$ ) in PBS was added to each well and incubated at $37^{\circ} \mathrm{C}$ for $4 \mathrm{~h}$, the supernatant was removed and replaced with $200 \mu \mathrm{L}$ of DMSO. The absorbance of the MTT formazan was determined at $570 \mathrm{~nm}$ in an Multimode Plate Reader (Perkin Elmer EnSpire ${ }^{\mathrm{TM}}$, Perkin Elmer, Inc.). The relative cell viability was expressed as a percentage of the control that was not treated with free lutein or LUT-NLP and PLL-LUT-NLP.

$$
\text { Cell proliferation }(\%)=\left(\mathrm{OD}_{\text {sample }} / \mathrm{OD}_{\text {control }}\right) \times 100 \%
$$

where, $\mathrm{OD}_{\text {control }}$ is the absorbance without sample at $570 \mathrm{~nm}$, and $A_{\text {sample }}$ is the absorbance that was treated with the lutein, LUT-NLP or PLL-LUT-NLP sample.

\subsection{Statistical analysis}

All measurements were replicated thrice. The experimental results were statistically tested for significance $(p \leq 0.05)$ for analysis of variance using SPSS (statistical product and service solutions) software. All data were expressed as the mean \pm standard deviation (SD).

\section{Results and discussion}

\subsection{Preparation and characterization of nanoliposomes}

3.1.1. Nanoliposomes entrapment efficiency (EE). Decorated nanoliposomes were prepared to investigate the effects of 0.04-0.08\% PLL on physical properties of LUT-NLP. As shown in Table 1, the EE of the decorated nanoliposomes increased in comparison with that of the undecorated ones. After being decorated with $0.06 \%(\mathrm{w} / \mathrm{v})$ PLL, the EE reached a higher value (up to $92.49 \%$ ). In the case of a higher decoration with PLL $(0.08 \%, w / v)$, the increase in EE was almost negligible, which might be due to an effect of absorption saturation for the electrostatic attraction between positive PLL and negative LUTNLP in the solution. On the other hand, the redundant PLL could not be combined with liposomes membrane due to the steric hindrance effect on a lower entrapment efficiency.

3.1.2. Particle size and zeta potential. All liposomes investigated were in the nanometer range. The particle size of LUTNLP was smaller than that of NLP due to the load of lutein. But the particle size of the decorated liposomes was slightly larger than that of the undecorated ones, which was dependent on PLL concentrations. Accompanied by PLL decoration, the particle size increased progressively. The particle size of undecorated nanoliposomes was $258.3 \pm 7.45 \mathrm{~nm}$ with a polydispersity index of $0.451 \pm 0.018$ (Fig. $2 \mathrm{~A}_{1}$, Table 1). Decorating the nanoliposomes with $0.04 \%$ PLL (w/v) increased hydrodynamic diameter of the nanoliposomes to $264.8 \pm 9.83 \mathrm{~nm}$ with a polydispersity index of $0.347 \pm 0.027$. It evidenced an interaction between PLL and LUT-NLP. A further increase in PLL concentration to $0.06 \%(\mathrm{w} / \mathrm{v})$ can be attributed to a significant increase in the liposomal size from $264.8 \pm 9.83 \mathrm{~nm}$ to $270.2 \pm$ $8.04 \mathrm{~nm}$ with a polydispersity index of $0.355 \pm 0.021$ (Fig. 2B $\mathrm{B}_{1}$, Table 1). Thus the adsorbed PLL on liposomal surface could lead to an increase of particle diameter. This might have

Table 1 Characterization of NLP, LUT-NLP and PLL-LUT-NLP: encapsulation efficiency (EE, \%), z-average diameter (nm), PDI and zeta potential

\begin{tabular}{|c|c|c|c|c|c|}
\hline Formulation & PLL (\%) & $\mathrm{EE}(\%)$ & $\mathrm{z}$-Average diameter (nm) & Polydispersity index (PDI) & Zeta potential $(\mathrm{mV})$ \\
\hline NLP & 0 & - & $436.2 \pm 7.34$ & $0.707 \pm 0.056$ & $-40.9 \pm 1.28$ \\
\hline LUT-NLP & 0 & $90.20 \pm 4.85$ & $258.3 \pm 7.45$ & $0.451 \pm 0.018$ & $-38.6 \pm 1.06$ \\
\hline PLL-LUT-NLP 1 & 0.04 & $91.81 \pm 5.21$ & $264.8 \pm 9.83$ & $0.347 \pm 0.027$ & $-34.3 \pm 0.91$ \\
\hline PLL-LUT-NLP 2 & 0.06 & $92.49 \pm 4.26$ & $270.2 \pm 8.04$ & $0.355 \pm 0.021$ & $-33.8 \pm 0.88$ \\
\hline PLL-LUT-NLP 3 & 0.08 & $92.93 \pm 5.39$ & $367.1 \pm 7.94$ & $0.400 \pm 0.036$ & $-27.9 \pm 0.68$ \\
\hline
\end{tabular}
$(\mathrm{mV})$. Data represent the mean value \pm standard deviation $(n=3)$ 
$\mathbf{A}_{1}$

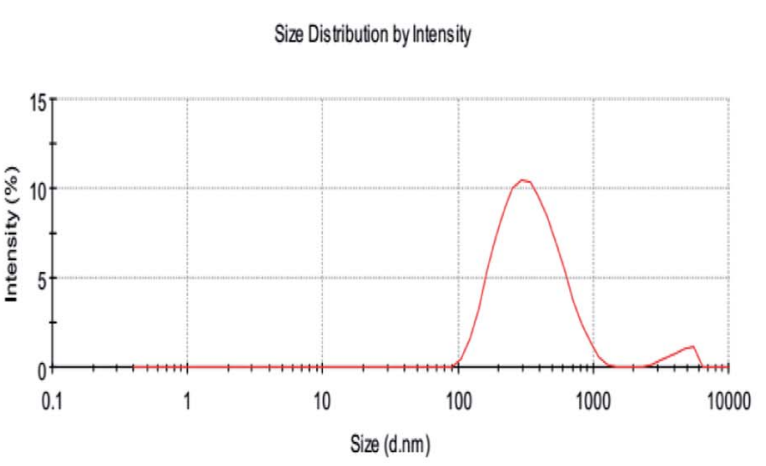

$\mathbf{B}_{1}$

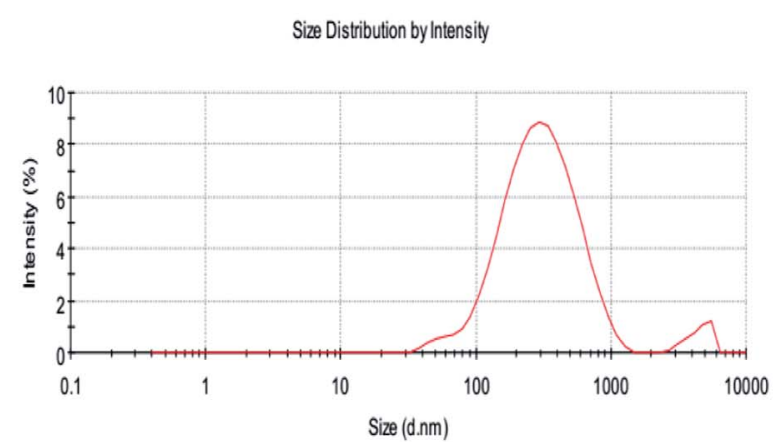

$\mathbf{A}_{2}$

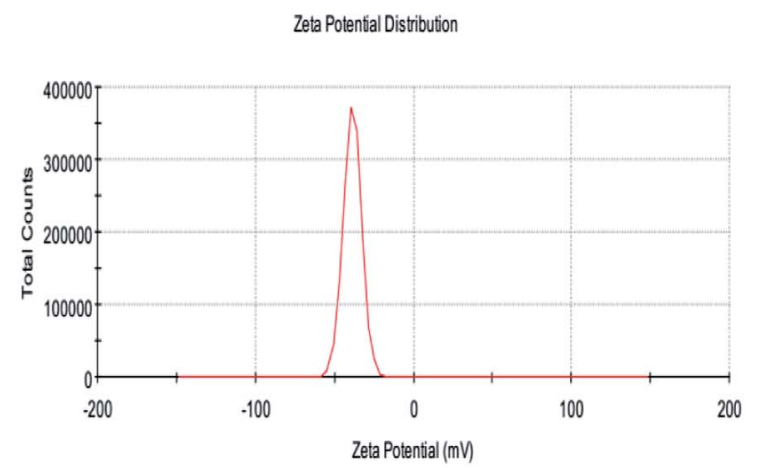

$\mathbf{B}_{2}$

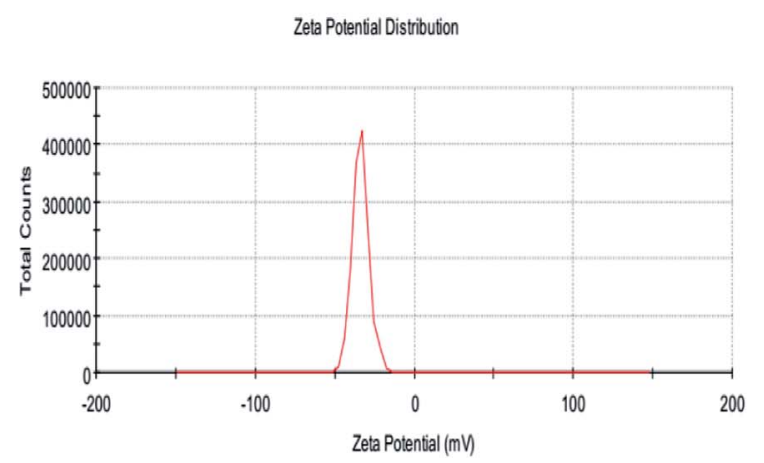

Fig. 2 Figure showing the average particle size $\left(A_{1}\right.$ and $\left.B_{1}\right)$ and zeta potential $\left(A_{2}\right.$ and $\left.B_{2}\right)$ of LUT-NLP (A), PLL-LUT-NLP (B) at the PLL concentration of $0.06 \%(\mathrm{w} / \mathrm{v}) \mathrm{PLL}$.

resulted from a further cross-link of the negatively charged nanoliposome by the positively charged PLL. However, upon addition of 0.08 (w/v) PLL, no significant change occurred in liposomal EE compared to that of $0.06 \%(w / v)$ PLL. It indicated that surface of nanoliposome was saturated with PLL. Therefore, the optimum concentration of PLL for the formation of its decorated nanoliposome was $0.06 \%(\mathrm{w} / \mathrm{v})$. Nanoliposomes size and polydispersity index (PDI) are important parameters. They directly refect their stability, biodistribution, and release of encapsulated compound.$^{40} \mathrm{PDI}$ values range from 0 to 0.5 , they are usually monodisperse and homogenous except those more than 0.5. ${ }^{41} \mathrm{PDI}$ of NLP was $0.707 \pm 0.056$, but it is lower in LUTNLP $(0.451 \pm 0.018)$ and PLL-LUT-NLP $(0.355 \pm 0.021)$ due to lutein load and the coated layers of PLL (Table 1), indicating a relatively homogeneous dispersion.

Zeta potential data showed that undecorated liposomes loading lutein possessed an lower average negative charge of $-38.6 \pm 1.06 \mathrm{mV}$ (Fig. $2 \mathrm{~A}_{2}$, Table 1). Small negative charge was attributed to the presence of phosphatidic acid in lecithin. ${ }^{42}$ After decorating with PLL at a concentration of $0.08 \mathrm{mg} \mathrm{mL}^{-1}$, there was a higher increase of LUT-NLP in zeta potential from $-38.6 \pm 1.06 \mathrm{mV}$ to $-27.9 \pm 0.68 \mathrm{mV}$ (Fig. $2 \mathrm{~B}_{2}$, Table 1 ). The increased zeta potential of PLL-LUT-NLP was ascribed to the amino group of PLL and the increase in charge suggested that the electrostatic interaction of positively charged polymer chains with the negative regions of the liposome surface was a major contribution of energy in the formation of the charged PLL. With a more PLL addition, the potential values increased continuously by an enhanced density of positive charge.

3.1.3. Transmission electron microscopy (TEM). TEM was used to attain the microstructure. As shown in Fig. 3, the morphology of LUT-NLP (Fig. 3A) and PLL-LUT-NLP (Fig. 3B) was spherical or oval with a bilayer membrane of nanoliposomes particles through self-assembly. The particle size ranged from 200 to $400 \mathrm{~nm}$. Due to the loaded lutein, a large proportion of lutein entrapped in the cores was globular, indicating that the lutein can be effectively incorporated into liposomal membrane. This could be attributed to the fact that lutein would well fit into the membrane architectures in a vertical manner due to its good lipid solubility. ${ }^{43}$ After decoration with PLL, the interaction of PLL with the surface of LUTNLP was well visualized. Compared to the LUT-NLP, PLL-LUTNLP exhibited more PLL aggregation absorbing on the membrane surface and surrounding LUT-NLP vesicle by the electrostatic adsorption. It formed a protective structure to improve the encapsulation efficiency of lutein and lead to an increase in particle size. The TEM results were in agreement with the particle size. It has been reported that PLL-modified nanoparticles could improve the biocompatibility and stability of drug loaded vesicle. ${ }^{44}$

3.1.4. FT-IR assay. FT-IR was used to further verify whether PLL was successfully decorated on lutein nanoliposome. FT-IR 
A

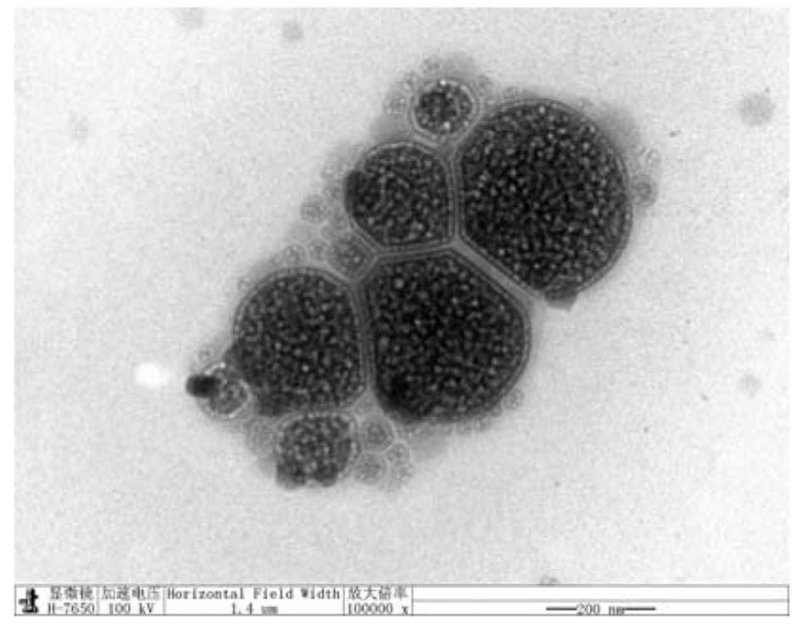

B

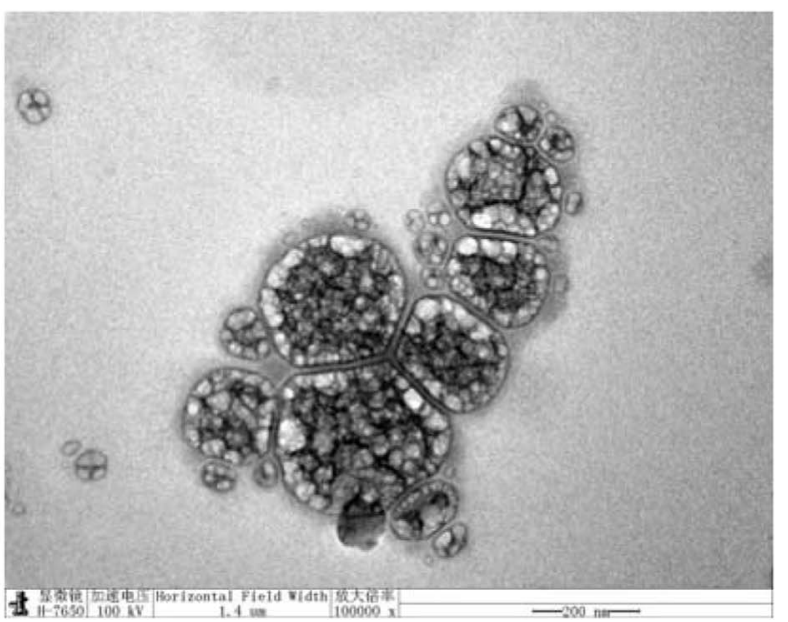

Fig. 3 TEM micrographs nanoliposomes: (A) undecorated liposomes (LUT-NLP), (B) 0.06\% (w/v) PLL decorated nanoliposomes (PLL-LUT-NLP).

spectrum of NLP, LUT-NLP and PLL-LUT-NLP are shown in Fig. 4, respectively. LUT-NLP showed its characteristic peaks of lecithin (vibration absorption of $\mathrm{CH}_{2}$ at $2923 \mathrm{~cm}^{-1}$ and $2857 \mathrm{~cm}^{-1}$ ), symmetrical stretching vibration absorption of $\mathrm{C}=\mathrm{O}\left(\right.$ at $\left.1735 \mathrm{~cm}^{-1}\right)$ stretching bands and $\mathrm{PO}_{4}\left(\right.$ at $\left.1248 \mathrm{~cm}^{-1}\right)$ antisymmetric stretching bands. ${ }^{45}$

When PLL was incorporated in nanoliposome, at the wavenumbers of $2923 \mathrm{~cm}^{-1}$ and $2857 \mathrm{~cm}^{-1}$ (vibration absorption of $\mathrm{CH}_{2}$ ), $1735 \mathrm{~cm}^{-1}$ (symmetrical stretching vibration absorption of $\mathrm{C}=\mathrm{O})$ and $1248 \mathrm{~cm}^{-1}\left(\mathrm{PO}_{4}\right.$ antisymmetric stretching bands) region, the location of peaks and form of PLL-LUT-NLP and LUT-NLP nanoliposome were similar.

PLL-LUT-NLP exhibited peaks at $1680-1403 \mathrm{~cm}^{-1}$ region (stretching vibration absorption of $\mathrm{C}=\mathrm{N}$ ). They were characteristic peaks of PLL and indicated an existence of PLL in PLL-LUT-NLP. FT-IR spectra of the decorated nanoliposome confirmed that LUTNLP had been successfully modified by PLL.

\subsection{In vitro digestion stability}

Degradation rates of free lutein, LUT-NLP and PLL-LUT-NLP in SGF and in SIF were estimated respectively to investigate the digestion stability in vitro. The remaining content of lutein was used to evaluate degradation rates of lutein in nanoliposome by taking lutein as a control group. Fig. 5 shows that a rapid degradation of lutein occurred when incubated in SGF and SIF after $12 \mathrm{~h}$. The free lutein degradation rates rose to $46.15 \pm$ 1.743 and $37.20 \pm 0.607$ after $12 \mathrm{~h}$, respectively.

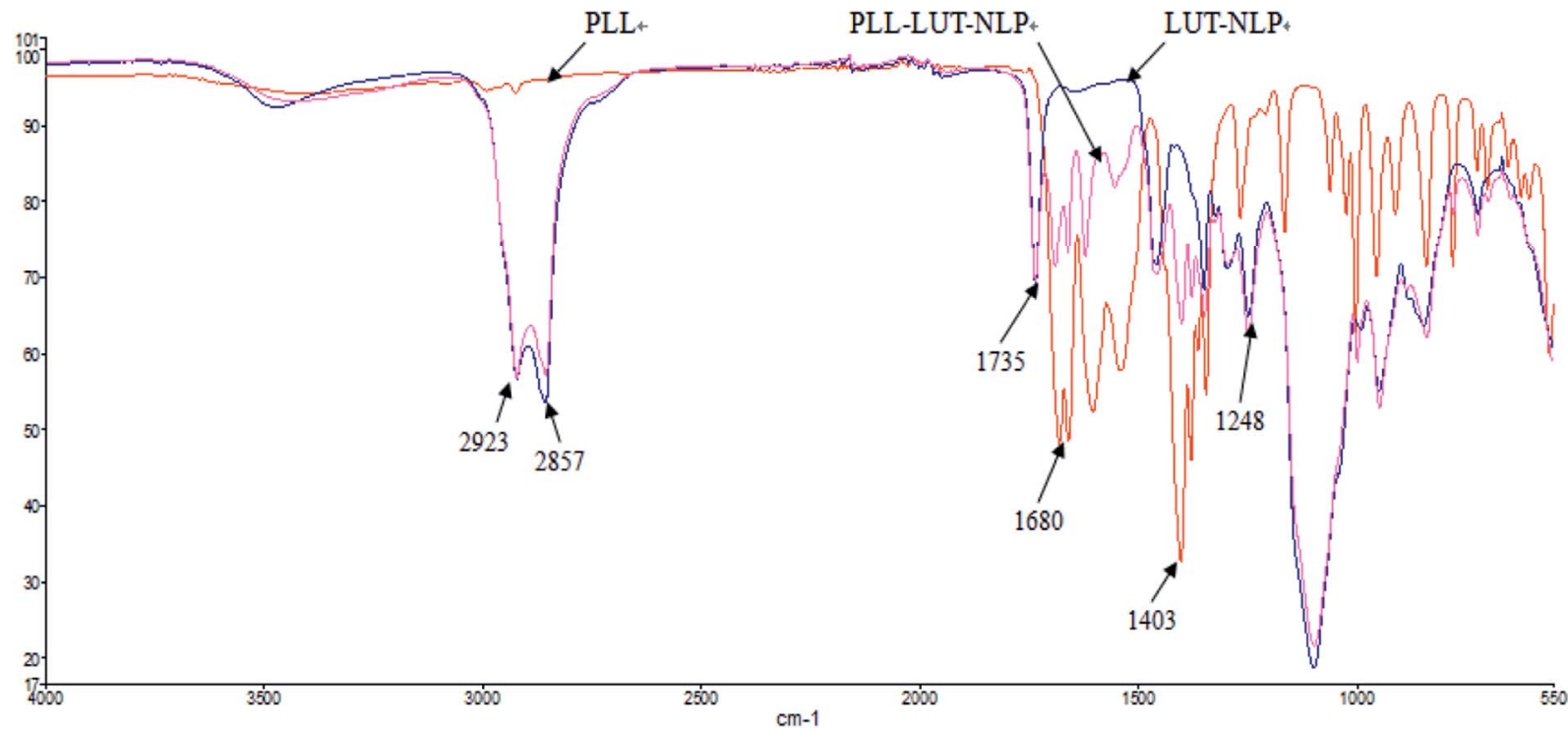

Fig. 4 FTIR spectra of PLL, undecorated LUT-NLP and PLL decorated PLL-LUT-NLP. 
A

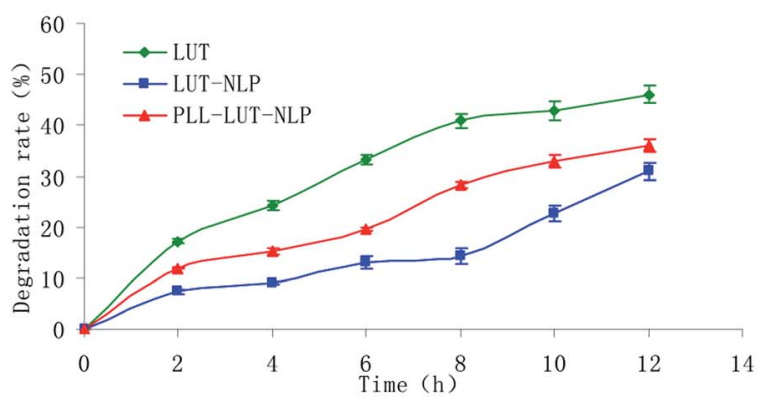

B

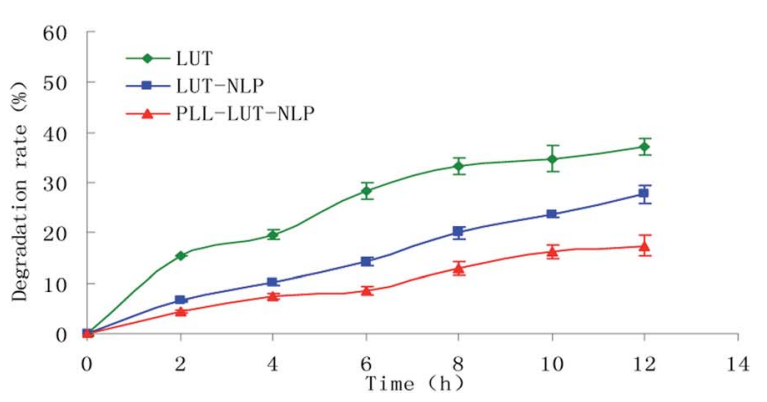

Fig. 5 Lutein degradation rate of free lutein, LUT-NLP, PLL-LUT-NLP during digestion in SGF (A) and in SIF (B) for 12 h.

Degradation of lutein was significantly reduced by encapsulating in NLP. The degradative lutein in LUT-NLP after incubating in SGF and SIF for $12 \mathrm{~h}$ were $30.95 \pm 0.321 \%$ and $27.67 \pm 0.80 \%$, respectively.

After decoration with PLL, lutein degradation rate dropped to $17.47 \pm 1.059 \%$ after $12 \mathrm{~h}$ incubation in SIF (Fig. 5B). But in SGF (Fig. 5A), it was $36.12 \pm 0.613 \%$, still higher than that in LUT-NLP. The results evidenced that hydrochloric acid in gastric juice and pepsase could weaken the interaction of PLL with the surface of LUT-NLP, and with the increased release of lutein from nanoliposome, the lutein degradation rates elevated slightly in SGF. When PLL aggregation was absorbed on the membrane surface, it could protect nanoliposome and thus reduce lutein degradation. The result verified that LUTNLP could decrease the degradation of lutein in SGF and in SIF, with and without PLL decoration, and increase the stability of lutein. LUT-NLP decorated with PLL could not increase the stability of lutein in SGF. However, PLL-LUT-NLP decreased the degradation rates of lutein in SIF (Fig. 5B). Therefore, it can be concluded that the stability of lutein in SGF and SIF is improved by encapsulation in nanoliposome. Furthermore, the lutein protective capability of PLL-LUT-NLP was much higher than that of nanoliposome in absence of PLL.

\subsection{In vitro release characteristics of LUT from nanoliposome}

The differences in lutein release between LUT-NLP and PLLLUT-NLP were performed for $20 \mathrm{~h}$ (longer than the intestinal digestion time) to verify the release characteristics. Sustained release of PLL-LUT-NLP was further evaluated by taking LUTNLP as control groups. Fig. 6 showed that LUT in nanoliposomes was released faster in SIF than in SGF. After $20 \mathrm{~h}$ incubation in SGF, LUT release rates for two of the samples were $43.28 \pm 2.16 \%$ and $51.26 \pm 3.33 \%$, respectively (Fig. 6A). However, after digestion in SIF, LUT release rate of LUT-NLP reached to $53.79 \pm 1.56 \%$ and that of PLL-LUT-NLP to 70.32 $\pm 1.42 \%$ (Fig. 6B). These facts indicate that PLL-LUT-NLP had a good sustainable release profile. LUT release rate in SGF and in SIF were higher than that of undecorated nanoliposomes. ${ }^{\mathbf{4 6}}$ Moreover, PLL-LUT-NLP released much more encapsulated lutein in SIF than in SGF and LUT release rate of PLL-LUT-NLP was much higher than LUT-NLP at 0-20 h in SIF. LUT-NLP released a similar proportion of lutein under both conditions.

This experiment has verified that PLL-LUT-NLP has a strong sustainable capacity for LUT release. It is a suitable delivery system for LUT. The possible reasons are that (1) the membrane permeability of PLL-LUT-NLP was increased by PLL decoration, while leakage of LUT from PLL-LUT-NLP was caused by reassembly of the membrane; and (2) the
A

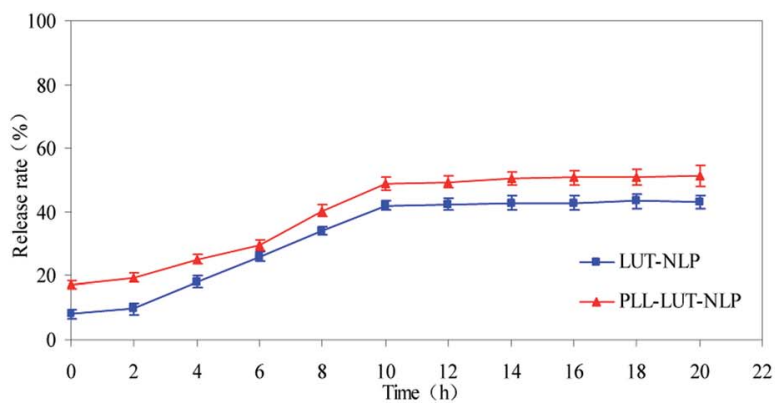

B

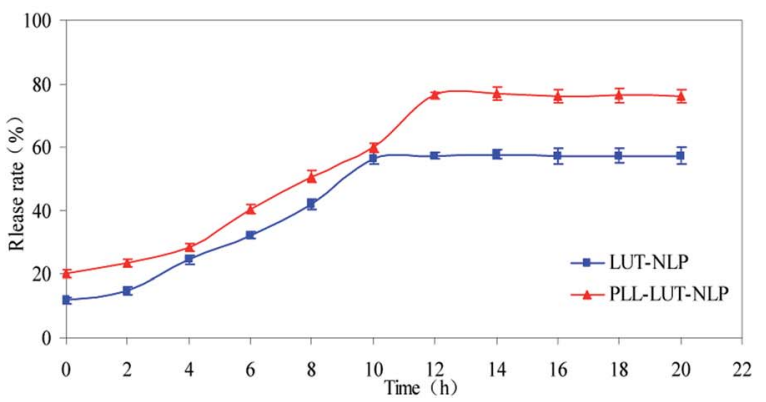

Fig. 6 Lutein release rate of LUT-NLP, PLL-LUT-NLP during digestion in SGF (A) and in SIF (B) for $20 \mathrm{~h}$. 
hydrotropic PLL molecules were adsorbed on the surface of the NLP and LUT entrapped near the surface. As the dissolution rate of the PLL near the surface is high, the amount of LUT released will be high as well.

\subsection{Determination of antioxidant activity}

3.4.1. DPPH free radical scavenging activity. The DPPH free radical scavenging capacity of the free lutein, LUT-NLP and PLL-LUT-NLP were tested based on their ability to scavenge the stable DPPH radical. As shown in Fig. 7A, the DPPH scavenging activity of free lutein was about $10.46 \%$ at $0.3 \mathrm{mg} \mathrm{mL}^{-1}$ lutein concentration, which is considerably lower than the value of
LUT-NLP (45.94\%) and PLL-LUT-NLP (46.8\%, 52.52\%, 56.22\%). There was no significant difference between radical scavenging activities of LUT-NLP and PLL-LUT-NLP when PLL decorating at lower concentrations (i.e. $0.04 \%$ ) resulted in lower scavenging activity. Although the addition of $0.06 \%$ and $0.08 \%(\mathrm{w} / \mathrm{v})$ PLL did not significantly affect the scavenging ability of nanoliposome containing lutein, a higher scavenging activity $(52.52 \%, 56.22 \%)$ was observed as compared with LUT-NLP (45.94\%). This may be attributed to antioxidant ability of lecithin in the nanoliposome, the DPPH scavenging activity of LUTNLP and PLL-LUT-NLP was higher than free lutein. When nanoliposome was decorated with PLL, more lutein could be

A

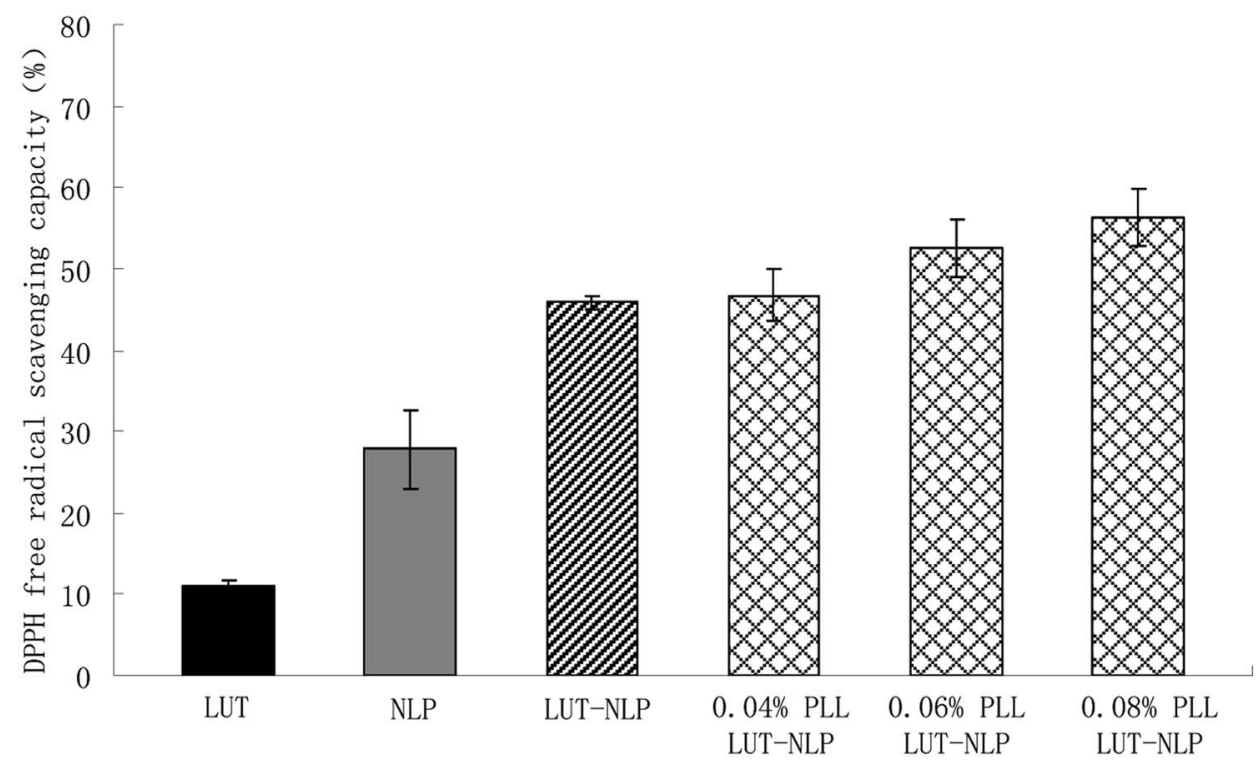

B

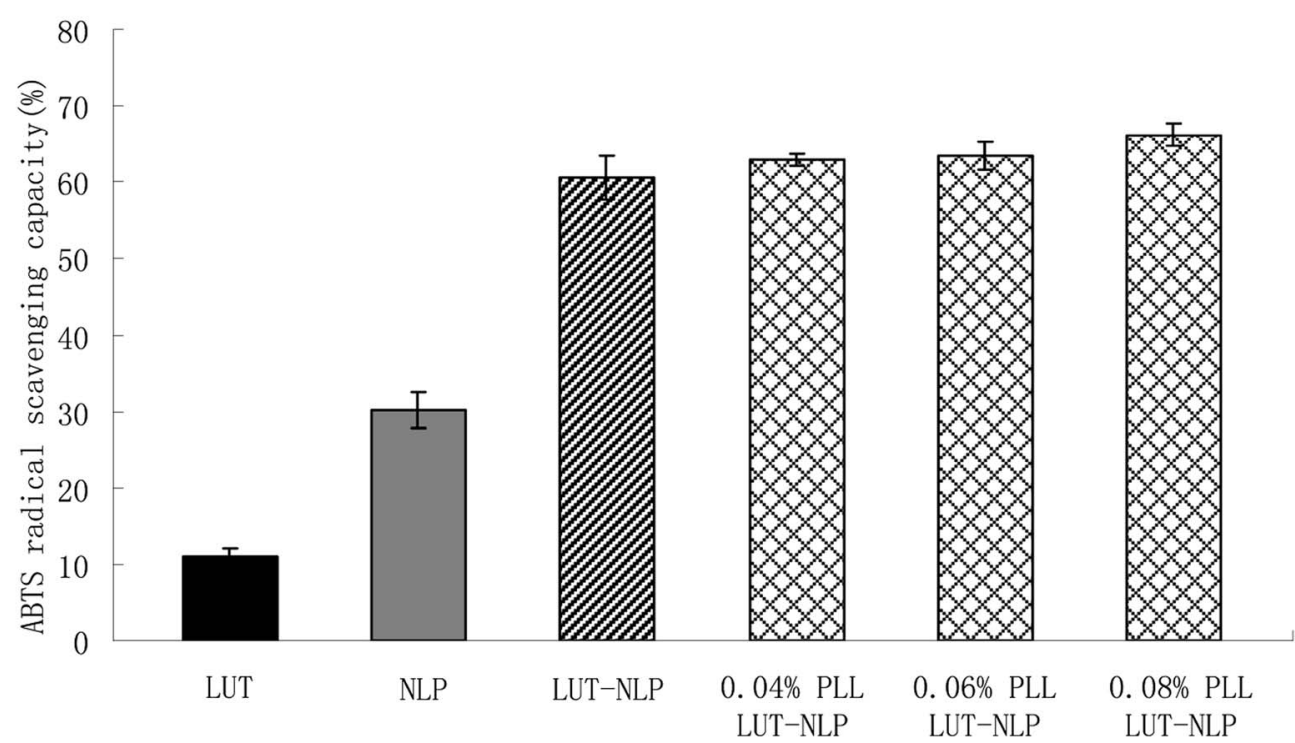

Fig. 7 (A) DPPH radical-scavenging capacity and (B) ABTS radical-scavenging capacity assay of the LUT, NLP, LUT-NLP and LUT-NLP decorated with PLL at different concentrations. 


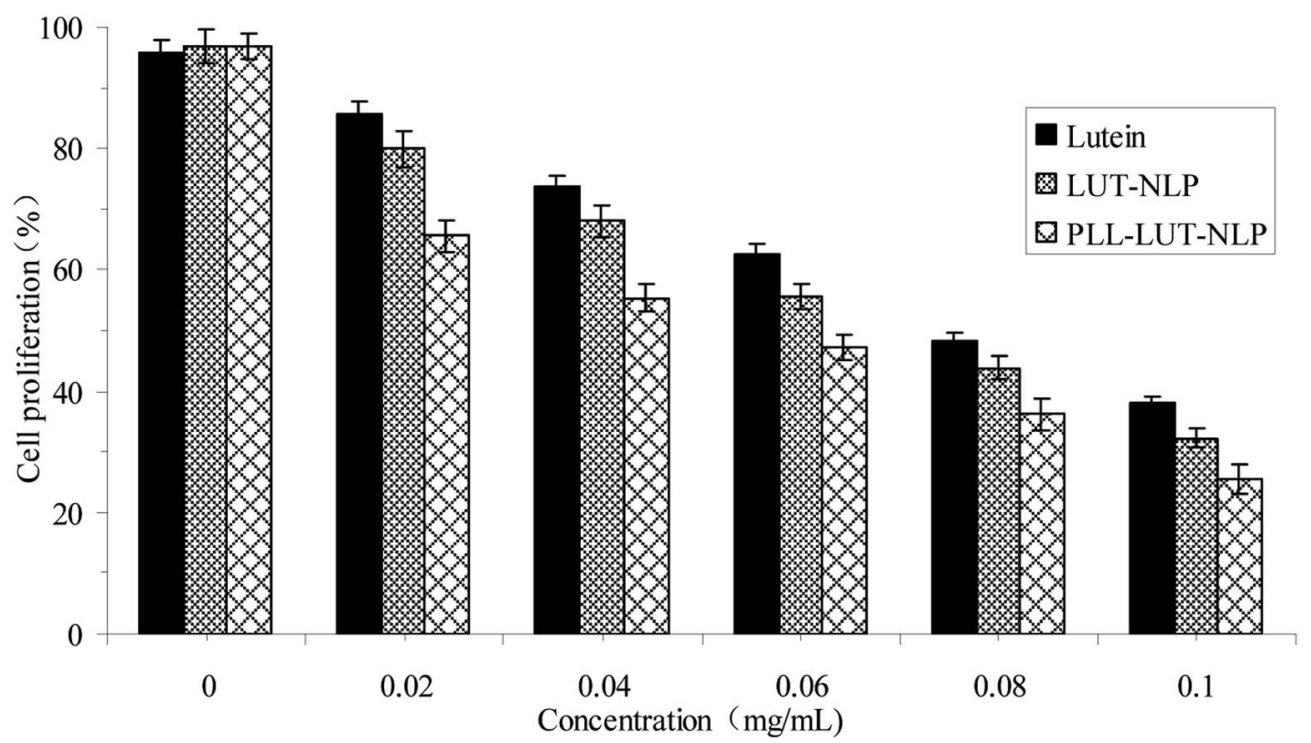

Fig. 8 Cell proliferation of Caco-2 cells treated with different concentrations of lutein, LUT-NLP and PLL-LUT-NLP $\left(0-0.1 \mathrm{mg} \mathrm{mL}^{-1}\right.$ lutein) for $24 \mathrm{~h}$.

released from PLL-LUT-NLP, the DPPH scavenging activity increased.

3.4.2. ABTS free radical scavenging activity. The antioxidant activity of the free lutein, evaluated by ABTS, was $11.1 \%$ (Fig. 7B), a significant increase in radical scavenging activity was observed after liposomal encapsulation. This may not only be attributed to antioxidant ability of lecithin in the nanoliposome, but also the higher solubility in aqueous phase of LUT-NLP and PLL-LUT-NLP. There are no significant differences between the LUT-NLP (62.88\%) and PLL-LUT-NLP (63.50\% and $66.20 \%$ ), this is in accordance with the result of DPPH assay. A slightly higher antioxidant capacity was observed after decorating with PLL.

3.4.3. Anti-proliferative activity. The MTT assay results demonstrated a concentration dependent activity after exposure to free lutein and nanoliposomes (Fig. 8). MTT assay was used to compare cell proliferation at different concentrations of lutein, LUT-NLP and PLL-LUT-NLP. The highest cell proliferations were observed in cells treated with free lutein. On the contrary, lower cell proliferations were observed in cells treated with LUTNLP and PLL-LUT-NLP, respectively. Compared to LUT-NLP, the PLL-LUT-NLP can induce higher cell apoptosis at different concentrations. At the end of the $24 \mathrm{~h}$ growth study, the cell proliferation in cells exposed to LUT-NLP was $55.56 \%$ higher than that of PLL-LUT-NLP at $0.06 \mathrm{mg} \mathrm{mL}^{-1}$ (Fig. 8) since the cell proliferation was $47.32 \%$ when the cells were exposed to PLL-LUT-NLP. These results demonstrate that PLL-decorated lutein nanoliposomes significantly inhibited tumor cell proliferation compared to lutein nanoliposomes. The reason may be that PLL has enhanced the cellular uptake of lutein by increasing the permeability of nanoliposomes and consequently inhibited the growth of Caco-2 cells. The mechanism for increasing or improving the bioactivity of PLL-decorated lutein nanoliposomes needs to be investigated further.

\section{Conclusions}

Our studies offer information about the physico-chemical properties of lutein nanoliposomes (LUT-NLP) and polypeptide-decorated lutein nanoliposomes (PLL-LUT-NLP). The increase in entrapment efficiency, size and zeta potential, as well as changes in morphology of PLL-LUT-NLP reflected several changes in the surface properties of nanoliposomes due to electrostatic attraction interactions. FTIR analysis showed that there were effective incorporated interactions between PLL and bilayer membrane of nanoliposomes. In addition, the performance of LUT was improved by NLP encapsulation during in vitro digestion. PLL-LUT-NLP decreased the degradation rates of lutein in SGF and in SIF, which showed a better stability in SIF than in SGF. Decoration of PLL on the surface of LUT-NLP efficiently promotes lutein release in vitro. Moreover, PLL-decorated nanoliposomes possessed the highest antioxidant activity compared with nanoliposomes and lutein, indicating that PLL-decorated liposomes protected lutein from oxidation and degradation in the external environment. The anticancer activity results showed that PLL-decorated nanoliposomes could inhibit proliferation of the Caco- 2 cells in a dose dependent manner.

These results suggest that PLL decorated nanoliposome system is a novel and effective delivery system for improving the physico-chemical properties (i.e. stability and release property) and bioactivity (i.e. antioxidant and antiproliferative) of lutein and other hydrophobic functional compounds, which may be quite interesting for food and pharmaceutical industry. Further investigations should be performed to focus on the cell uptake and absorption mechanism of PLL-decorated lutein nanoliposome on molecular level. 


\section{Conflicts of interest}

The authors declare that this article content has no conflict of interest.

\section{Acknowledgements}

This research was financially supported by Jiangsu Planned Projects for Postdoctoral Research Funds (No. 1601028A) and by the University Nursing Program for Young Scholars with Creative Talents in Heilong jiang Province (No. UNPYSCT-2016091).

\section{References}

1 A. Kamil, D. E. Smith, J. B. Blumberg, C. Astete, C. Sabliov and C. Y. Oliver Chen, Food Chem., 2016, 192, 915-923.

2 A. Sujak, J. Gabrielska, W. Grudziński, R. Borc, P. Mazurek and W. I. Gruszecki, Arch. Biochem. Biophys., 1999, 371, 301-307.

3 E. S. M. Abdel-Aal and I. Rabalski, Am. J. Biomed. Sci., 2013, 5, 109-125.

4 N. I. Krinsky, J. T. Landrum and R. A. Bone, Annu. Rev. Nutr., 2003, 23, 171-201.

5 D. C. Musch, JAMA Ophthalmol., 2014, 132, 139-141.

6 I. D. Nwachukwu, C. C. Udenigwe and R. E. Aluko, Trends Food Sci. Technol., 2016, 49, 74-84.

7 Y. Li, Y. Zhang, X. Liu, M. Wang, P. Wang, J. Yang and S. Zhang, Int. J. Oncol., 2018, 52, 2119-2129.

8 R. L. Luan, P. C. Wang, M. X. Yan and J. Chen, Eur. Rev. Med. Pharmacol. Sci., 2018, 22, 1514-1520.

9 T. Narisawa, Y. Fukaura, M. Hasebe, M. Ito, R. Aizawa, M. Murakoshi, S. Uemurab, F. Khachik and H. Nishino, Cancer Lett., 1996, 107, 137-142.

10 R. Arunkumar, K. V. H. Prashanth and V. Baskaran, Food Chem., 2013, 141, 327-337.

11 S. Takagi, T. Yamagami, K. Takeda and T. Takagi, Agric. Biol. Chem., 1987, 51, 1567-1572.

12 A. Murugeshu, C. Astete, C. Leonardi, T. Morgan and C. M. Sabliov, Nanomedicine, 2011, 6, 1513-1528.

13 D. Y. Hong, J. S. Lee and H. G. Lee, Int. J. Biol. Macromol., 2015, 85, 9-15.

14 M. I. Adamczak, E. Hagesaether, G. Smistad and M. Hiorth, Int. J. Pharm., 2016, 498, 225-233.

15 M. R. Mozafari, Methods Mol. Biol., 2010, 605, 29-50.

16 C. Tan, B. Feng, X. Zhang, W. Xia and S. Xia, Food Hydrocolloids, 2016, 52, 774-784.

17 A. Junghans, H. Sies and W. Stahl, Arch. Biochem. Biophys., 2001, 391, 160-164.

18 W. Liu, M. Tian, Y. Kong, J. Lu, N. Li and J. Han, LWT-Food Sci. Technol., 2016, 75, 608-615.

19 M. Murata, K. Nakano, K. Tahara, Y. Tozuka and H. Takeuchi, Eur. J. Pharm. Biopharm., 2012, 80, 340-346.

20 A. Makhlof, S. Fujimoto, Y. Tozuka and H. Takeuchi, Eur. J. Pharm. Biopharm., 2011, 77, 216-224.

21 K. Tahara, S. Furukawa, H. Yamamoto and Y. Kawashima, Int. J. Pharm., 2010, 392, 311-313.
22 D. Volodkin, V. Ball, P. Schaaf, J. C. Voegel and H. Mohwald, BBA, Biochim. Biophys. Acta, Biomembr., 2007, 1768, 280-290.

23 D. Volodkin, H. Mohwald, J. C. Voegel and V. Ball, J. Controlled Release, 2007, 117, 111-120.

24 H. Sasaki, K. Karasawa, K. Hironaka, K. Tahara, Y. Tozuka and H. Takeuchi, Eur. J. Pharm. Biopharm., 2012, 83, 364369.

25 S. H. Kim, J. H. Jeong, K. W. Chun and T. G. Park, Langmuir, 2005, 21, 8852-8857.

26 C. Lu, X. Li, W. Q. Xia, S. H. Lu, H. J. Luo, D. Y. Ye, Y. M. Zang and D. J. Liu, Acta Biomater., 2017, 49, 434-443.

27 G. H. Shin, S. K. Chung, J. T. Kim, H. J. Joung and H. J. Park, J. Agric. Food Chem., 2013, 61, 11119-11126.

28 C. Sebaaly, H. Greige-Gerges, S. Stainmesse, H. Fessi and C. Charcosset, Food Biosci., 2016, 15, 1-10.

29 C. Tan, S. Xia, J. Xue, J. Xie, B. Feng and X. Zhang, J. Agric. Food Chem., 2013, 61, 8175-8184.

30 D. J. Li, J. F. Song and C. Q. Liu, Int. J. Food Prop., 2015, 18, 178-185.

31 F. S. Juveriya, I. Fathima, V. Abhishek and F. Khanum, Food Chem., 2016, 197, 562-570.

32 C. Tan, J. Xue, X. Lou, X. W. Lou, S. Abbas, Y. Guan, B. Feng, X. M. Zhang and S. Q. Xia, Food Funct., 2014, 5, 1232-1240.

33 L. Q. Zou, W. Liu, W. L. Liu, R. H. Liang, T. Li, C. M. Liu, Y. L. Cao, J. Niu and Z. Liu, J. Agric. Food Chem., 2014, 62, 934-941.

34 G. Davidov-Pardo, C. E. Gumus and D. J. Mcclements, Food Chem., 2016, 196, 821-827.

35 M. Frenzel and A. Steffen-Heins, Food Chem., 2015, 173, 1090-1099.

36 M. Gibis, C. Ruedt and J. Weiss, Food Res. Int., 2016, 88, 105113.

37 K. Papoutsis, P. Pristijono, J. B. Golding, C. E. Stathopoulos, M. C. Bowyer, C. J. Scarlett and Q. V. Vuong, J. Food Process. Preserv., 2017, 41, 1-8.

38 L. Ramezanzade, S. F. Hosseini and M. Nikkhah, Food Chem., 2017, 234, 220-229.

39 A. Sousa, P. Araújo, J. Azevedo, L. Cruz, I. Fernandes, N. Mateus and V. de Freitas, Food Chem., 2016, 192, 142-148.

40 M. Reza Mozafari, C. Johnson, S. Hatziantoniou and C. Demetzos, J. Liposome Res., 2008, 18, 309-327.

41 H. K. Park, J. K. Yoon and K. Kim, Langmuir., 2006, 22, 16261629.

42 S. J. Choi, E. A. Decker, L. Henson, L. M. Popplewell, H. Xiao and D. J. Mcclements, Food Res. Int., 2011, 44, 3006-3012.

43 Q. Lu, P. M. Lu, J. H. Piao, X. L. Xu, J. Chen, L. Zhu and J. G. Jiang, LWT-Food Sci. Technol., 2014, 57, 686-695.

44 X. Wang, F. Wei, A. Liu, L. Wang, J. C. Wang, L. Ren, W. M. Liu, Q. Tu, L. Li and J. Y. Wang, Biomaterials, 2012, 33, 3719-3732.

45 L. Q. Zou, S. F. Peng, W. Liu, L. Gan, W. L. Liu, R. H. Liang, C. M. Liu, J. Niu, Y. L. Cao, Z. Liu and X. Chen, Food Res. Int., 2014, 64, 492-499.

46 T. Chuacharoen and C. M. Sabliov, Colloids Surf., A, 2016, 503, 11-18. 